\title{
Formulation and Storage of Platelet-Rich Plasma Homemade Product
}

\author{
Olivier Bausset, ${ }^{1}$ Laurent Giraudo, ${ }^{1}$ Julie Veran, ${ }^{1}$ Jeremy Magalon, ${ }^{1}$ Jean-Marie Coudreuse, ${ }^{2}$ Guy Magalon, ${ }^{3}$ \\ Christophe Dubois, ${ }^{4}$ Nicolas Serratrice, ${ }^{3}$ Françoise Dignat-George,${ }^{5}$ and Florence Sabatier ${ }^{1}$
}

\begin{abstract}
The platelet-rich plasma (PRP) is an autologous biotherapy based on platelet-healing properties. Here, we developed a simple and reproducible PRP purification protocol based on two successive centrifugations. We evaluated different centrifugation speeds and time-storage durations on the platelet quantity and quality. Sterility and stability of our PRP homemade product were also performed. We prepared PRP from 54 healthy volunteers. We tested activation state, reactivity, and stability of platelets by flow cytometry using basal and adenosine diphosphate (ADP)-induced P-selectin expression markers; growth factor release after platelet activation by an enzymelinked immunosorbent assay (ELISA); platelet aggregation capacity by aggregrometry assays; clot formation and retraction by thromboelastography; and platelet morphology by ultrastructural analysis. About 130 and $250 \mathrm{~g}$ successive speed centrifugations further concentrated platelets while preserving their bioactivity during $6 \mathrm{~h}$ (after that, platelet functions were significantly altered). In these conditions, we obtained a highly concentrated pure PRP product (with a low leukocyte count) suitable to study platelet properties. To avoid the loss of efficacy, we recommend injecting PRP under $3 \mathrm{~h}$ after preparation.
\end{abstract}

\section{Introduction}

$\mathbf{T}$ HE CONCEPT OF platelet-rich plasma (PRP) for healing stimulation has been existed for over 25 years now. Briefly, platelets, once activated, release bioactive proteins (growth factors and cytokines) that improve reparative and regenerative processes. PRP is basically purified by gentle centrifugation from whole peripheral blood. It is easy, fast, safe (due to its autologous origin), and cheap. PRP is commonly used in various medical fields, as rheumatology, orthopedic, regenerative sport medicine, and plastic surgery, with remarkable results.

Since 2000, thousands of papers have recently been published about PRP, so that there are many papers trying to characterize and classify the numerous techniques available on the market in terms of preparation (centrifugation speed and use of anticoagulant), content (platelets, leucocytes, and growth factors), and applications. Currently, all these aspects are strongly discussed in the literature, and there is no con- sensus. Moreover, many authors do not clearly know the characteristics of the product they are using, creating many misunderstandings.

Previously, many authors tried to determine what would be the best concentrations and protocols for several clinical applications. Finally, there is no evidence of standardization of PRP preparation and use. Different methods of preparation may produce different platelet concentrations, and each preparation may produce different products with different applications. For this reason, the development of autologous formulations with a controlled platelet counting and consequently, a controlled bioactive factor release appears challenging.

Here, we propose to collect some information about the quantity and quality of platelets from PRP after different centrifugation speed and duration time storage before platelet physiology alteration. Again, there is no best speed for PRP preparation; there are only speeds leading to different products. To do that, we developed a protocol based on two

This work was performed in the Cell Therapy Unit, University Hospital La Conception, Marseille, France

${ }^{1}$ Cell Therapy Unit, ${ }^{3}$ Department of Plastic Surgery, and ${ }^{5}$ Hematology Laboratory, CHU Conception, Marseille, France.

${ }^{2}$ Sport Medicine Unit, CHU Salvator, Marseille, France.

${ }^{4}$ INSERM, UMR911, Centre de Recherche en Oncologie Biologique et Oncopharmacologie, Marseille, France. 
successive centrifugations. A first spin, also called soft spin, separates plasma, buffy coat (containing PRP), and cells. A second spin or hard spin, further concentrates platelets. In these conditions, we obtained highly concentrated platelets from whole blood. To preserve platelet functionality and reactivity during centrifugation and storage conditions, we determined the PRP product quality by in vitro testing of the activation/reactivity status and growth factor release activity.

It could be interesting to get some intact platelets, although several studies pointed that PRP's gathering significant quantities of leukocytes was also useful, for example, in chronic elbow tendinopathy. ${ }^{1-3}$ Currently, there is no established correlation between growth factor release and residual white blood cells in PRP. The content (growth factors, platelets, and circulating cells) ${ }^{4}$ is determining for these products, and several experts in different sport medicine fields highlighted better clinical results when higher cell concentrations were used with very limited quantities of leukocytes.

\section{Material and Methods}

\section{Whole-blood source}

Fifty-four healthy volunteer donors (33 men and 21 women) included 22-59-year-olds (mean 38.18 years), with no relevant diseases and free of any drugs known to affect platelet functions for 7 days before the study. To maintain platelet integrity, we used preferentially the citrate anticoagulant. Approximately $34 \mathrm{~mL}$ of whole blood collected in four 10-mL tubes (Vacuette ${ }^{\circledR}$, Ref. 455001; Greiner Bio-One) with $1.5 \mathrm{~mL}$ of adenosin citrate dextrose-acid (ACD-A) solution (Fenwal, Ref. BDB8651) makes $40 \mathrm{~mL}$ for each volunteer. ACD-A anticoagulant is the most frequently used for PRP preparation. Indeed, ACD-A better maintains the intraplatelet signal transduction mechanisms during PRP preparation, thus improving the overall responsiveness of platelets. ${ }^{5,6}$

Another tube, coated with the ethylenediaminetetraacetic acid anticoagulant, was used to determine the platelet number and concentration with an automatic cell counter (Advia 2120 ${ }^{\circledR}$; Siemens Diagnostic Solutions). All donors included in this study had platelet numbers over 150,000 platelets/mL.

\section{PRP production}

First spin (soft spin) was done with a standard laboratory centrifuge (Thermo Scientific) at $130 \mathrm{~g}$ during $15 \mathrm{~min}$. We obtained two different phases: a first clouded phase containing platelets, platelet-poor plasma (or PPP), and buffy coat, and a second phase containing red blood cells, representing, respectively $45 \%$ and $55 \%$. Some authors directly collect the buffy coat phase with a needle, but this technique did not guarantee an optimal platelet number. Thus, we preconize to collect the clouded phase directly by gentle aspiration with a pipette (approximately $2 \mathrm{~mL} /$ tube). This product was generally contaminated by red blood cells from the pellet. To further concentrate PRP, we proceeded to a second $15-$ min spin in four new 11-mL conic tubes (NUNC, Ref. 56423). Different centrifugation speed conditions were tested (130, 250, 400, and $1000 \mathrm{~g}$ ). Supernatants (or PPP) were removed by gentle aspiration (approximately $1 \mathrm{~mL} /$ tube). Then, pellets containing platelet were resuspended in the re- sidual plasma and pooled. Finally, we obtained a total of $4 \mathrm{~mL}$ of concentrated PRP. About $250 \mu \mathrm{L}$ sample was used to determine $\mathrm{pH}$, platelet, and leukocyte numbers. About $100 \mu \mathrm{L}$ sample was injected in the pediatric blood culture bottle (BacT/ALERT ${ }^{\circledR}$ device; bioMérieux) for sterility testing. All manipulations for PRP preparation and sampling were conducted under a class A microbiological safety facility located in the cell therapy unit at the Conception Hospital (Assistance Publique des Hôpitaux de Marseilles, France). Under these conditions, we obtained a controlled cellular therapy product, optimized for human medical applications.

\section{Growth factor quantification assays}

Growth factor release was assayed after platelet activation using the method previously described by Anitua et al. ${ }^{4}$ PRP samples were transferred in glass tubes and activated by calcium chloride $(\mathrm{CaCl}): 50 \mu \mathrm{L}$ of $\mathrm{CaCl} 10 \%$ for $1 \mathrm{~mL}$ of PRP ratio. Matrices were allowed to retract for $30 \mathrm{~min}$ at $37^{\circ} \mathrm{C}$, and then supernatants were collected by gentle aspiration and stored at $-80^{\circ} \mathrm{C}$ until quantification of growth factors vascular endothelial growth factor (VEGF), platelet-derived growth factor (PDGF-AB), transforming growth factor (TGF- $\beta 1$ ), and insulinlike growth factor (IGF-1) by enzyme-linked immunosorbent assay (ELISA) kits (R\&D Diagnostics). Assays were performed in duplicates according to the manufacturer's instructions.

\section{Platelet concentration and $\mathrm{pH}$ quality control}

Several in vitro assays were conducted to evaluate the quality of platelets after purification and storage. The $\mathrm{pH}$ of the PRP product was measured using litmus paper strips.

\section{Flow cytometer analyses}

Platelet activation and reactivity were determined by flow cytometry after P-selectin expression in basal conditions or incubated with $200 \mu \mathrm{M}$ of adenosine diphosphate (ADP is an agonist) for $5 \mathrm{~min}$ at room temperature (RT). PRP was detected by a CD62P monoclonal antibody labeled with fluorescein isothiocyanate (FITC) (clone LYP20; Biocytex) or using an FITC-conjugated isotype control antibody (clone 2DNP2H11; Biocytex). After $30 \mathrm{~min}$ of incubation, platelets were resuspended in $500 \mu \mathrm{L}$ of appropriate saline buffer and analyzed with an FC-500 analyzer (Beckman Coulter). Light scatter and fluorescence channels were set up at logarithmic gain and platelets gated according to forward- and side-scatter properties. P-selectin-positive expression was detected over a nonspecific background signal from isotype control labeling. A total of 5000 events monitored using CXP software (Beckman Coulter) were used for data processing. Results were expressed as the ratio of mean fluorescence intensity (MFI) for the specific labeling of the entire platelet populations with control antibody labeling/over MFI associated to an irrelevant isotype control labeling.

\section{Aggregometry}

The aggregometry assay evaluated the platelet aggregation capacity. Cartridges for single use (Laborgeräte $\mathrm{GmbH}$ ) are placed into the aggregometer (APACT-4; Helena Biosciences Europe) and filled with $270 \mu \mathrm{L}$ of PRP samples, which were obtained after the second spin (130 and $1000 \mathrm{~g})$, and adjusted for platelet counting of $250 \mathrm{~g} / \mathrm{L}$ by dilution in a patient's PPP. 
About $30 \mu \mathrm{L}$ of $100 \mu \mathrm{M}$ ADP (Helena Biosciences Europe) was added to initiate aggregation. PRP was maintained at $37^{\circ} \mathrm{C}$ with stirring and light transmission, recorded during $240 \mathrm{~s}$, and later analyzed using a software program (APACT Software 1.0; PASS Engineering). Three hundred microliters of PPP was used as negative control to check platelet autoaggregation.

\section{Thromboelastography}

Thromboelastography determined the PRP capacity to support clot formation and retraction as previously described, ${ }^{7,8}$ using a TEG-500 instrument (Haemoscope Corporation). Eight hundred forty microliters of PPP and $60 \mu \mathrm{L}$ of PRP were obtained after second spin (250 and $400 \mathrm{~g}$ ) and transferred to a vial containing buffered stabilizers and Kaolin $^{\circledR}$ (phospholipids). Samples were mixed by gentle inversion five times and transferred to a $37^{\circ} \mathrm{C}$ prewarmed disposable cup containing $20 \mu \mathrm{L}$ of $\mathrm{CaCl}$. The rigidity of the clot composed of platelet aggregates and fibrin strands was monitored over a 40-min period time. The parameters used for the $\mathrm{TEG}^{\circledR}$ analysis included time to initiate fibrin formation (R), time to clot formation (k), and maximum amplitude (MA). Data analyses were performed using integrated software (TEG software). Regarding analysis of platelet concentrations, a short R, a short $\mathrm{k}$, and a high MA demonstrate a good reaction of platelets, their ability to initiate a clot, and also their activation. ${ }^{9}$

\section{Electron microscopy}

To visualize centrifugation speed effects on platelet morphology, ultrastructural analyses of platelets were performed by transmission electron microscopy (TEM) as previously described. ${ }^{10}$ After the second spin, platelet pellets were resuspended and washed twice in a $0.1 \mathrm{M}$ cacodylate buffer, fixed with $2.5 \%$ glutaraldehyde in a $0.1 \mathrm{M}$ cacodylate buffer ( $\mathrm{pH} 7.2$ ), and postfixed in osmium tetroxide $2 \%$ in the same buffer. Pellets were dehydrated in graded alcohol solutions and embedded in an EMbed-812 kit. Ultrathin sections $(60 \mathrm{~nm})$ were counterstained with uranyl acetate and lead citrate before observation with a TEM (JEM-1400; JEOL Ltd.) at an $80-\mathrm{kV}$ accelerating voltage. Pseudopodium formation and granule repartition were morphological criteria used to assess the platelet activation status.

\section{PRP stability}

Flow cytometer analyses of P-selectin expression and growth factor measurement were performed on the PRP product obtained from five healthy donors from the cohort (130 and $250 \mathrm{~g}$ successive spins) and maintained up to $24 \mathrm{~h}$ at RT.

\section{Statistical analysis}

GraphPad Prism 5.0 software was used for statistical analyses. A $p$-value of $<0.05$ was considered as significant. All quantitative measurements were described using summary statistics ( $n$, mean, standard deviation). The Mann-Whitney test was used to analyze the possible influence of the second speed spin on the platelet counting and functionality of these preparations. To compare the mean of the four groups unmatched, an analysis of variance (ANOVA) followed by the
Kruskal-Wallis nonparametric test was conducted. The Spearman's rank correlation coefficient (rS) was used to demonstrate the relationship between PRP thrombocyte and leukocyte counting and PRP growth factor levels.

\section{Results}

\section{Determination of optimal conditions for PRP purification}

Platelet concentration. The mean platelet counting in donors' whole blood was $260 \pm 38 \mathrm{~g} / \mathrm{L}$. PRP preparation based on a second centrifugation speed of 130-1000 $g$ resulted in platelet concentration, since platelets counts were significantly higher in each condition, compared to the starting whole-blood material (Fig. 1A). The mean platelet concentration factor defined as the platelet count in PRP compared to the platelet count in whole blood ranged from 3.01 to 3.96, with a significant difference between centrifugation protocols (Fig. 1B). The platelet concentration factor was significantly higher with $250 \mathrm{~g}$ speed centrifugation compared to $130 \mathrm{~g}$ ( $3.47 \pm 0.35$ and $3.01 \pm 0.30$, respectively, $p=0.02$ ). However, a centrifugation performed at 400 or $1000 \mathrm{~g}$ did not further increase the platelet concentration factor compared to a $250 \mathrm{~g}$ centrifugation (3.73 $\pm 0.80, p=0.64$ and $3.96 \pm 0.62, p=0.14$, respectively). Yet, for $36 \%$ of PRP preparations obtained with $1000 \mathrm{~g}$ centrifugation $(n=4 / 11)$, we encountered more difficulties to resuspend platelet pellets. This was probably due to platelet aggregation, indicating that high-spin centrifugation could be deleterious for platelet function and is not appropriate for PRP preparation.

Platelet aggregation capacity. Platelets aggregation assays indicated that various centrifugation speeds did not significantly impact on the aggregation rate induced by ADP (Fig. 2). A trend toward a higher aggregation rate was significant with the lower centrifugation speed $(130 \mathrm{~g})$ compared to other conditions. In addition, we observed that a higher speed $(1000 \mathrm{~g})$ induced spontaneous autoaggregation of platelets in $36 \%$ of cases compared to lower centrifugation speeds.

Ultrastructure of platelets. TEM analyses showed that higher centrifugation speeds considerably modified the platelet morphology during PRP preparation. At $130 \mathrm{~g}$ centrifugation speed, platelets displayed a discoid form with low number of pseudopodia. Internal organization was characterized by numerous granules, dense bodies, mitochondria, and glycogen particles randomly distributed throughout the cytoplasm (representative image in Fig. 3A). These features are consistent with the resting platelet-known morphology. ${ }^{10}$ After ADP treatment (used as positive control), platelet morphological changes was observed such as spheroid shape, extension of numerous pseudopodia, and centralization of granules (representative image in Fig. 3D left panel). These morphological changes are consistent with an activated state of platelets. ${ }^{10,11}$ In addition, some small-sized aggregates composed by closely packed platelets could be visualized (Fig. 3D right panel). With lower-speed centrifugation, we also noted that there are less morphological activation indicators than in ADP-activated platelets. At 250 and $400 \mathrm{~g}$ (Fig. 3B, C respectively), we observed a high number of pseudopodia and a trend toward centralization of granules. Taken together, these observations indicated that lower centrifugation speeds were better for the resting platelet morphology preservation. 

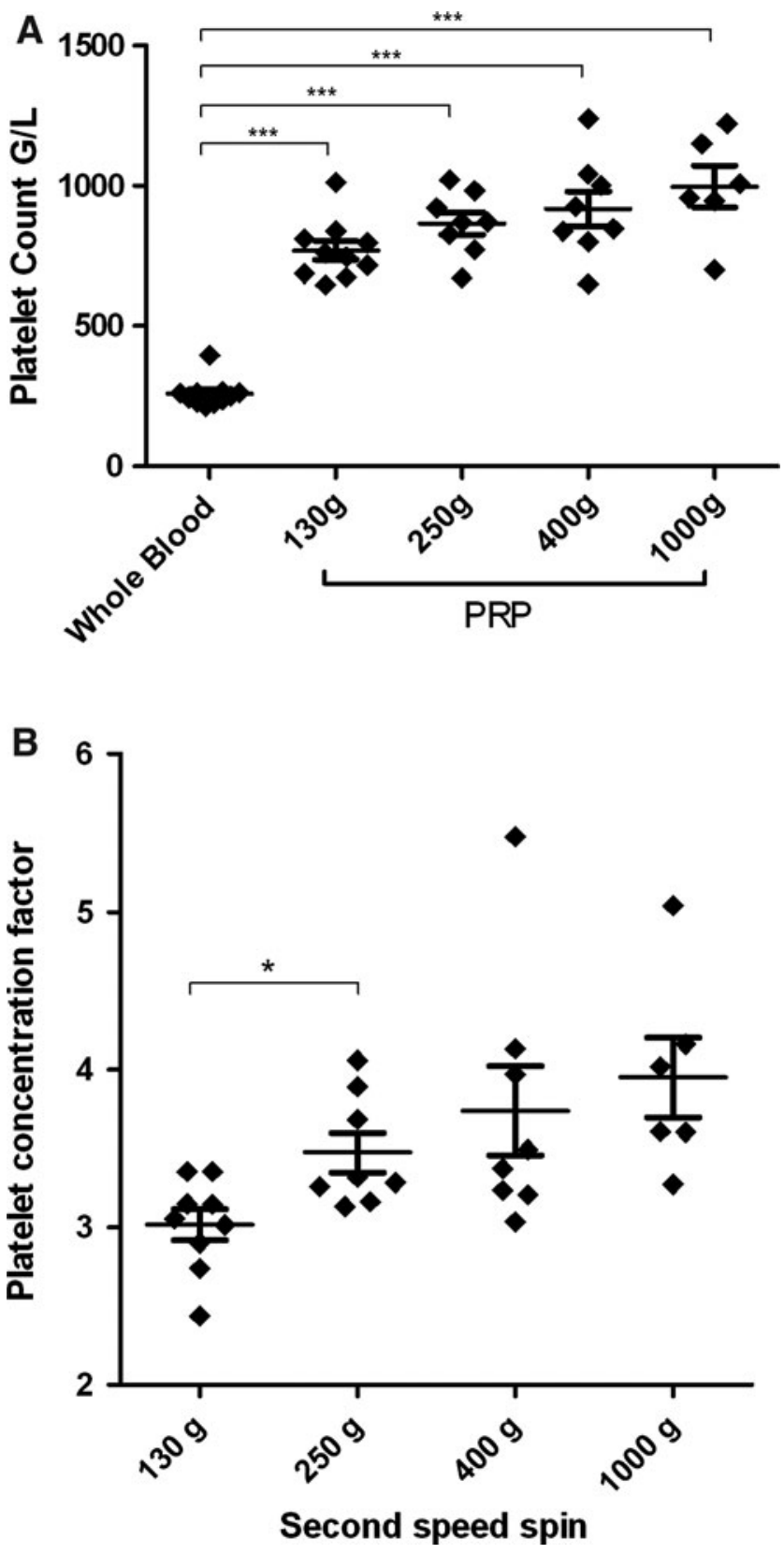

FIG. 1. (A) Platelet counting in whole blood and in four conditions of PRP preparation. Data obtained for different donors (130 g, $n=11 ; 250 g, n=10 ; 400 g, n=10 ; 1000 g, n=6)$. *** $p<0.0003$ comparing with whole blood. (B) Platelet concentration factor versus speed of second centrifugation. Data obtained for different donors (130 $g, n=10 ; 250 g, n=8 ; 400 g$, $n=8 ; 1000 g, n=6)$. ${ }^{*} p=0.02$. PRP, platelet-rich plasma.

PRP-induced clot formation. Consistent with aggregation tests, thromboelastography analyses showed no significant difference in thromboelastography (TEG) parameters between PRP prepared at 250 and $400 \mathrm{~g}$ (Fig. 4), although the time to clot formation tended to be higher at $250 \mathrm{~g}$ compared to $400 \mathrm{~g}$. These results indicated that second spin centrifugation $<400 \mathrm{~g}$ maintained the procoagulant activity of platelets.

Flow cytometry. Basal P-selectin expression and ADPinduced P-selectin expression were measured by flow cytom-

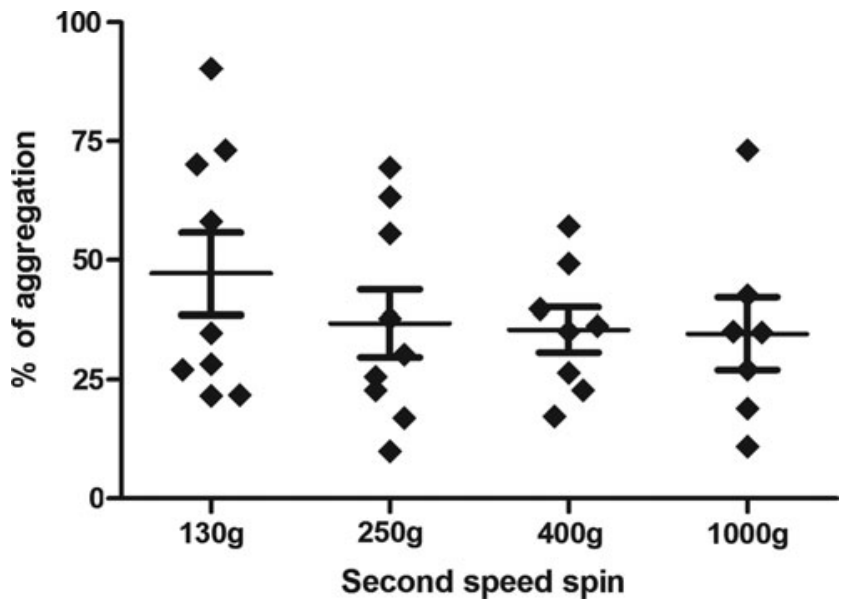

FIG. 2. Aggregation rate versus speed of the second centrifugation. Data obtained for different donors (130 $g, n=10$; $250 g, n=8 ; 400 g, n=8 ; 1000 g, n=6)$.

etry to evaluate, respectively, the impact of centrifugation protocols on the activation state and reactivity of platelets. In basal conditions, membrane expression of P-selectin remained very low after PRP preparation, and no significant difference could be detected in the P-selectin expression level between the various conditions of the centrifugation speed tested (Fig. 5). ADP induced P-selectin expression of platelets significantly differed between the various tested centrifugation protocols (ANOVA test, $p=0.0022$ ). Indeed, the ADPinduced P-selectin expression decreased when the speed of the second centrifugation increased. These results indicate that a higher centrifugation speed is associated with impaired platelet reactivity toward agonist.

Collectively, these results evidenced that (1) a 1000-g centrifugation speed induced platelet aggregation in vitro, decreased platelet reactivity to agonist as shown by flow cytometry assessment of ADP-induced P-selectin expression, and morphological changes consistent with platelet activation; (2) a 250-g centrifugation speed is associated with a slight improvement of platelet reactivity to agonist and minor morphological changes compared to a 400- $g$ centrifugation spin while the platelet concentration factor is similar; (3) a 130-g protocol is associated with platelet preservation and functions, but has a significant decrease in platelet concentration compared to $250 \mathrm{~g}$. Therefore, we selected the $250-g$ centrifugation speed for further experiments in view to investigate PRP growth factor release.

\section{Growth factor release activity and influence of storage duration}

Measurement of growth factor levels. To evaluate the capacity of PRP to release growth factors, ELISA assays were performed after in vitro platelets activation. Mean levels of VEGF, PDGF-AB, and TGF- $\beta 1$ were significantly higher in PRP compared to PPP (Fig. 6A). No significant difference was observed in IGF-1 levels between PRP and PPP. PDGF$\mathrm{AB}$ release by PRP was found to be significantly correlated to the platelet count (Spearman $r, \mathrm{rS}=0.55$ ) (Fig. 6B). Such relationship was not significant for TGF- $\beta 1\left(R^{2}=0.35\right)$ and VEGF levels $(\mathrm{rS}=-0.34)$. No correlation could be established 

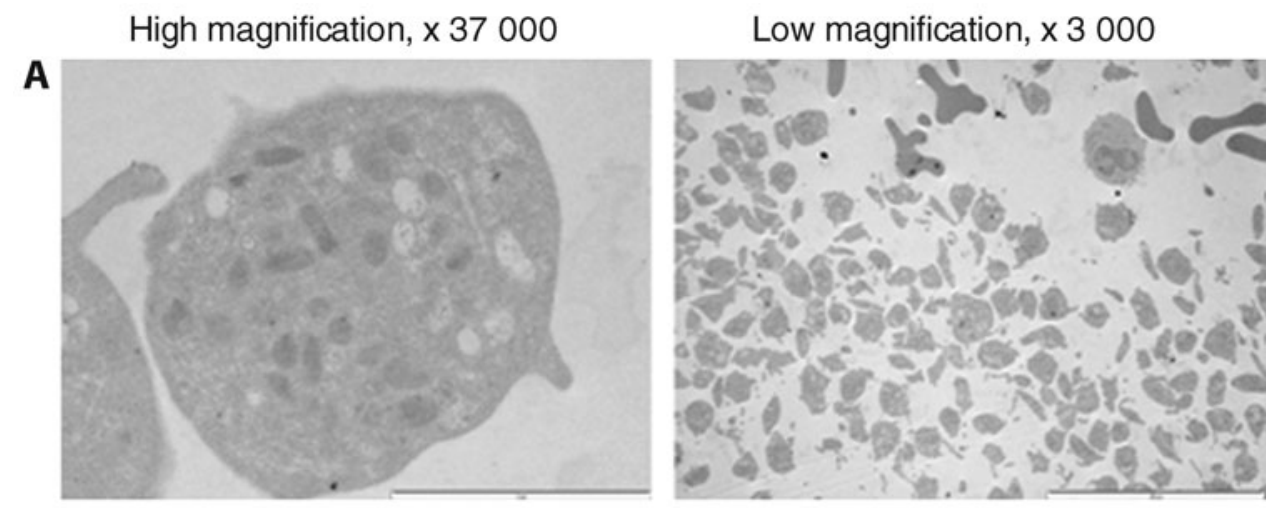

B

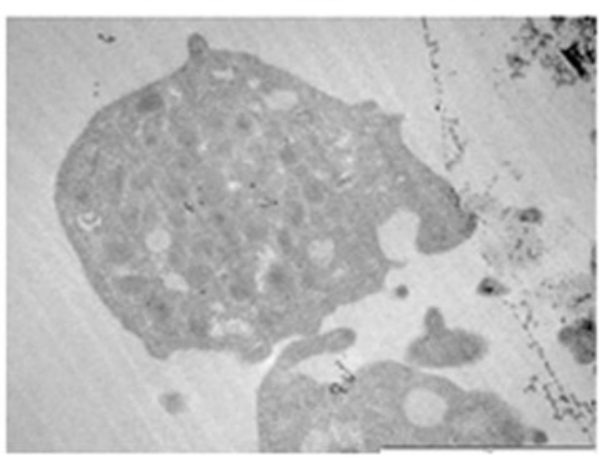

C
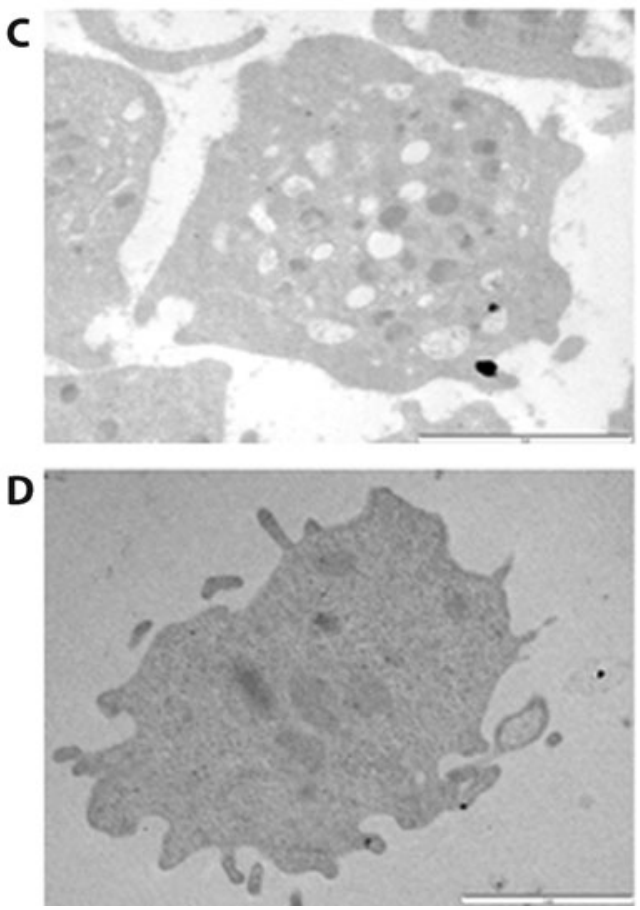
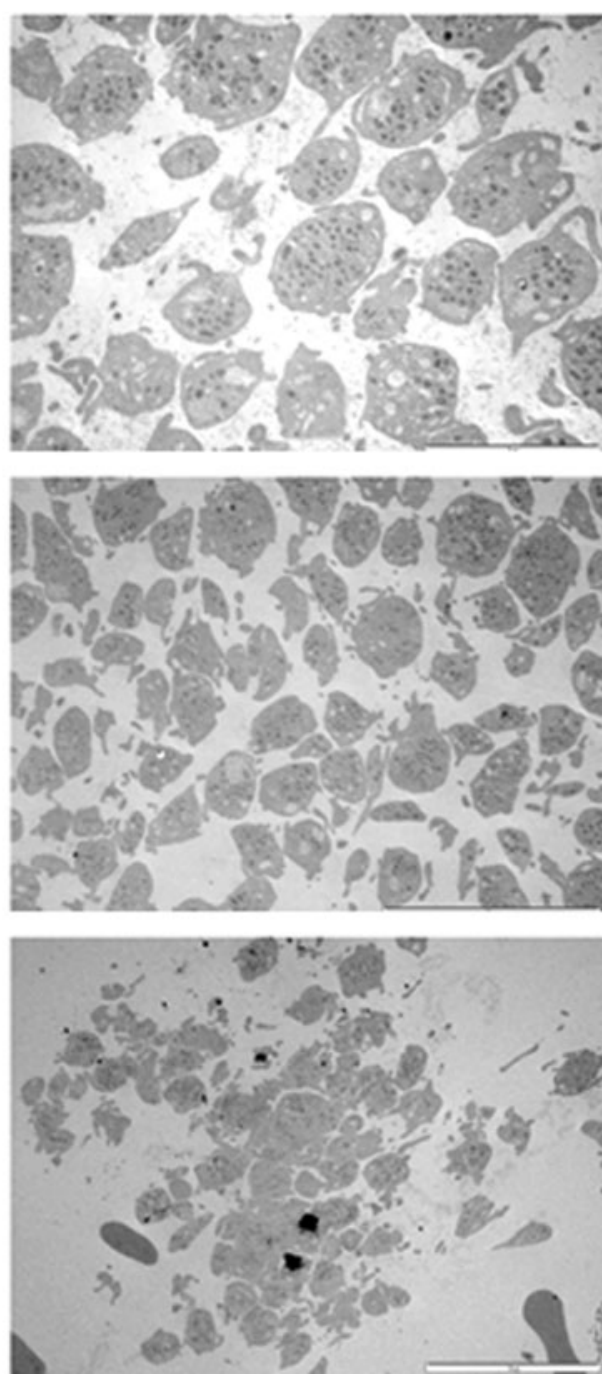

FIG. 3. Ultrastructure of platelet issued of one PRP preparation and analyzed by transmission electron microscopy. Centrifugation protocol (A) $130 \mathrm{~g}$; (B) $250 \mathrm{~g}$; (C) $400 g$, and (D) positive control induce by ADP. (A) Typical section of a resting platelet, discoid forms without pseudopodia, with well-preserved granular structure (panel left). Note the formation of loose aggregates in (A) and (B) (right panel) with largely preserved granular structure and nonactivated platelets. (D) Typical section corresponding to morphological signs of activation: spherical forms with pseudopodia, a partial centralization of a-granules, and some large aggregates composed of mostly degranulated platelets. ADP, adenosine diphosphate. between growth factor release and residual leukocytes counted in PRP.

PRP stability. Flow cytometry analyses were performed to evaluate the activation status and reactivity toward PRP agonist stored up to $24 \mathrm{~h}$ after preparation. PRP storage was associated to an increase in platelet membrane basal levels of P-selectin that reached statistical significance $24 \mathrm{~h}$ after the end of PRP preparation (MFI signal/isotypic 2.14 \pm 0.50 vs. 9.26 $\pm 3.79, p=0.079$ ) (Fig. 7). A reduction of ADP-induced upregulation of P-selectin expression was also observed over time as evidenced by a significant difference in P-selectin membrane levels at $6 \mathrm{~h}$ compared to the value at the end of PRP preparation (MFI signal/isotypic 17.98 \pm 4.23 vs. $12.09 \pm 1.09 p=0.0079$ ) (Fig. 7). These data indicate that a 6-h storage duration induced platelet activation and alteration of response to the agonist.

Three PRP samples were used to evaluate the growth factor-releasing capacity immediately after purification and after storage duration of 3,6 , and $24 \mathrm{~h}$ at $20^{\circ} \mathrm{C}$. VEGF and PDGF- 
$\mathbf{k}$

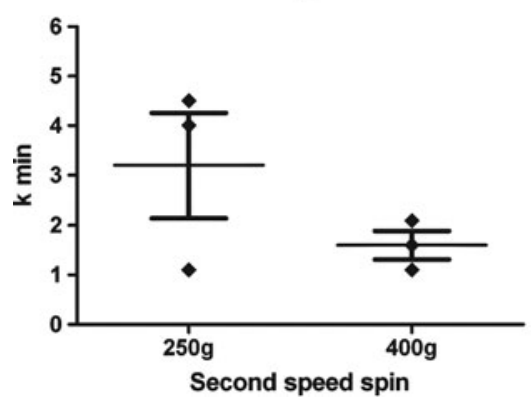

MA

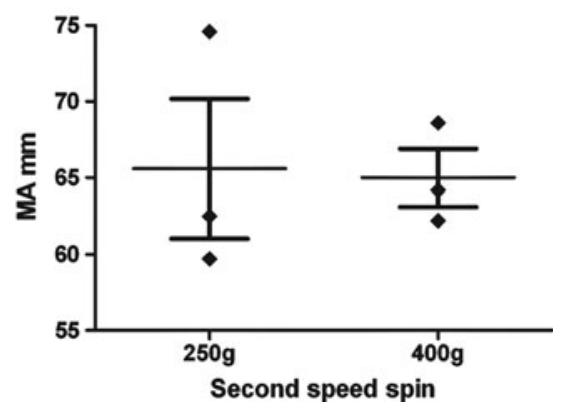

FIG. 4. Thromboelastrography results. Time to clot formation (k) and maximum amplitude (MA). Results from three independent PRP preparations.
Sterility, leukocyte counting, and $\mathrm{pH}$ measurement. Microbiological analyses of all PRP preparations inoculated in BacT/ALERT blood culture bottles showed the absence of microbial growth after 10 days of incubation, indicating that the donor blood sampling and PPR preparation process ensured maintenance of product sterility. Mean leukocyte counting was $3.8 \pm 0.44 \mathrm{~g} / \mathrm{L}$ (second spin speed $250 \mathrm{~g}, n=13)$, and the mean of $\mathrm{pH}$ measured on 10 samples of PRP was $7.6 \pm 0.45$.

\section{Discussion}

This study proposes a standardized method for PRP preparation based on the formulation of the obtained biotherapy product. We evidenced that a simple protocol, including two serial 15-min centrifugations of anticoagulated whole blood at 130 and $250 \mathrm{~g}$ successively, allows an appropriate enrichment of platelets with preservation of platelet functionality and growth factor release capacity. Knowledge of these biological characteristics forms the basis for clinical studies allowing establishment of the relationship between PRP specifications and clinical impact.

The growing number of articles being published on PRP applications have brought about clinical benefits. Nevertheless, no studies provided information about PRP biological qualification, neither using PRP from commercially available kits nor using PRP from a homemade laboratory method. The lack of characterization of these different available PRP products makes the literature very difficult to interpret. Without a consensus, this therapeutic field remains opaque, and its development is considerably hampered. ${ }^{12}$ Many studies are limited by the unknown amount of platelets and the unknown quantity of growth factors present in the injected PRP. ${ }^{13}$ As a result, in the different proposed indications of PRP, levels of factors required for clinical efficacy are currently undetermined.

Cho et al. ${ }^{14}$ reported that the platelet amount should be three to seven times higher than the basal value. According to Jungbluth et al., ${ }^{15}$ a platelet concentration three to five times higher than in peripheral blood appears to be particularly advantageous to stimulate bone regeneration. Below this concentration, the effect of PRP is suboptimal, and paradoxically, a higher concentration can inhibit bone regeneration. ${ }^{15}$ Consistently, in our experiments, a mean platelet concentration factor of 3.47 can be achieved using a protocol based on a second centrifugation performed at $250 \mathrm{~g}$, indicating that increasing higher centrifugation speed may not be necessary for clinical efficacy.
FIG. 5. P-selectin expression versus speed of the second centrifugation. Data obtained for different five donors. ${ }^{* *} p<0.008$ and ${ }^{*} p<0.016$. Signal $/$ isotypic $=$ P-selectin expression (MFI) of basal or ADP-induced condition/MFI of isotypic control in arbitrary units. MFI, mean fluorescence intensity. 
A
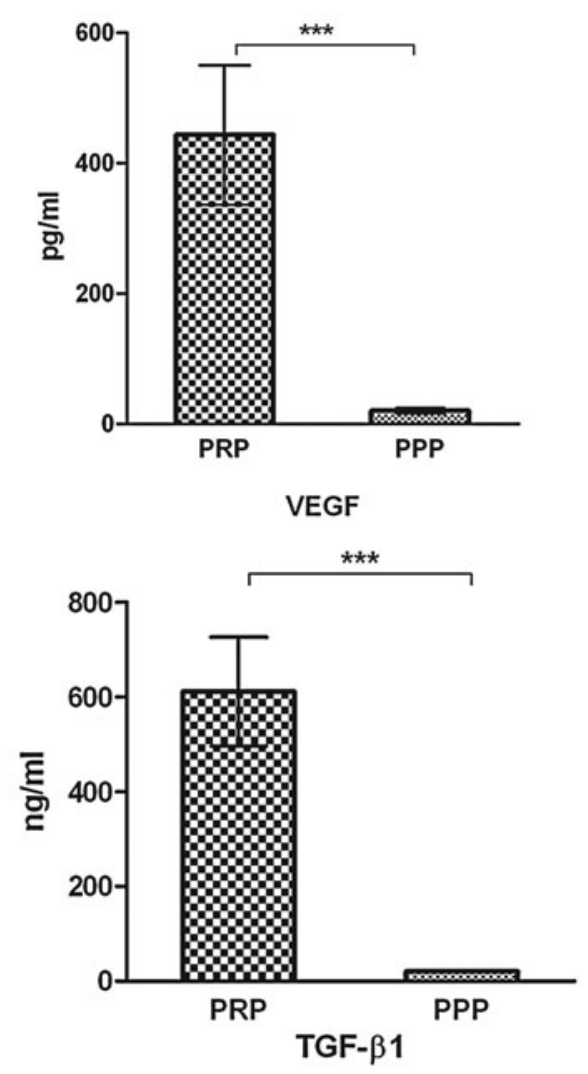
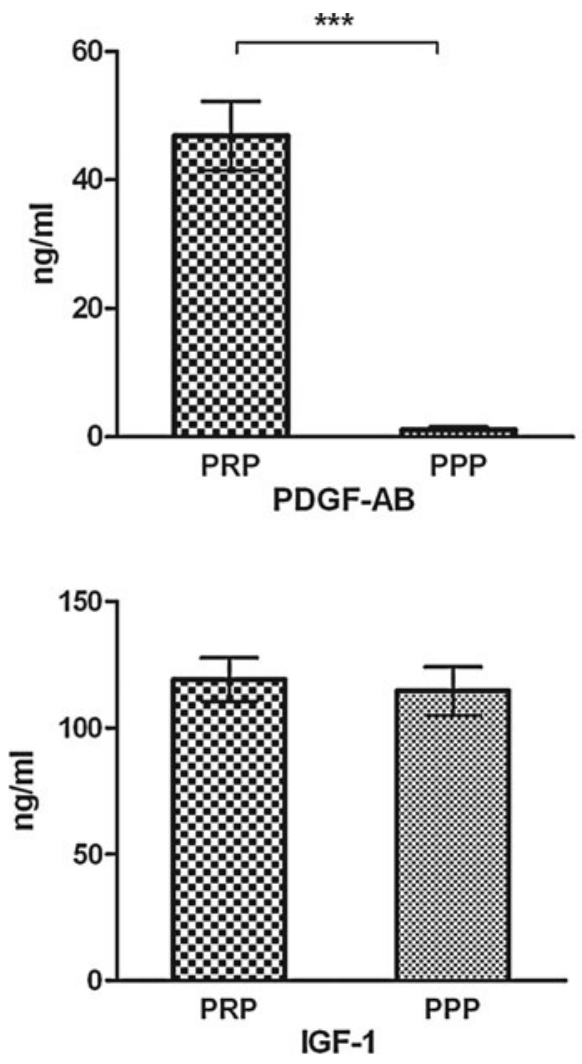
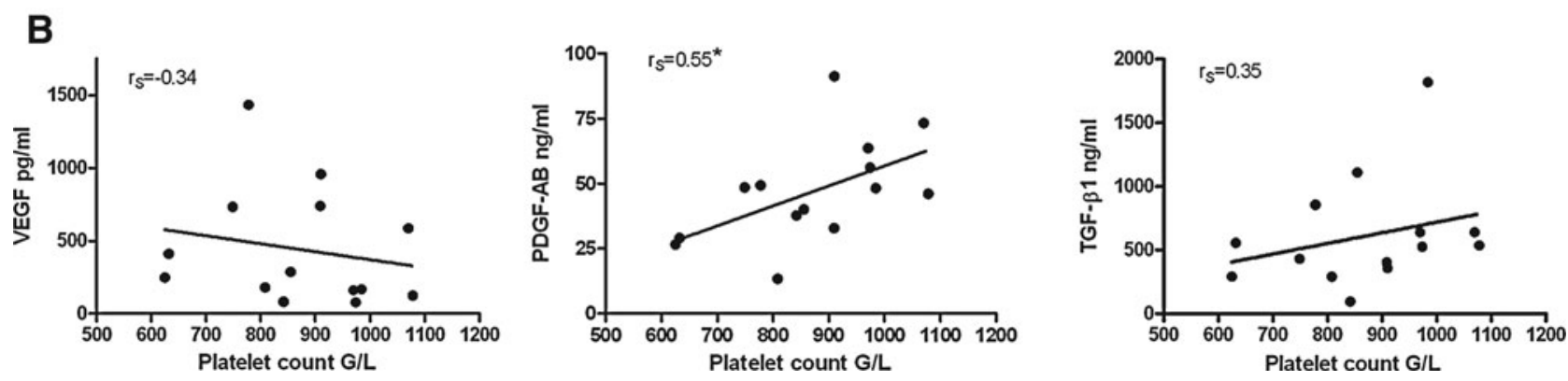

FIG. 6. (A) Concentrations of growth factors in two different plasma preparations. PPP and PRP (enriched in platelets 3.47fold over peripheral blood). Data obtained for different 14 donors. ${ }^{* * *} p<0.0001$. (B) Scatter plot of the PRP growth factor levels versus platelet count. Data obtained for different 14 donors. *Statistically significant. PPP, platelet-poor plasma; rS, Spearman's rank correlation coefficient.

Alteration of platelets functionality was shown during preparation and storage of platelets. ${ }^{8,9}$ In addition, a complementary analysis of platelet quality enables us to confirm that a higher centrifugation speed may severely alter platelet functionality. This fact was evidenced by the occurrence of platelet autoaggregation, reduction of platelet response to agonist, and transition from a resting to an activated morphology visualized by electron microscopy. Although aggregometry and TEG assays did not reveal significant differences in the platelet behavior between the different second speed spins tested, an alteration of the P-selectin upregulation in presence of ADP was observed at 400 - and $1000-g$ protocols. Such alteration has been recognized as a reliable marker of platelet hyporesponsiveness to agonist, with a predictive value for in vivo functionality of platelet. ${ }^{16}$ Therefore, this result mainly contributed to identify the $250-g$ centrifugation protocol as the optimal condition for PRP preparation. Again, Landesberg et al. ${ }^{17}$ evaluated the effect of different centrifugation forces and showed that $>800-g$ spin may reduce the amount of growth factor released by PRP.

The actual challenge for PRP optimization is to determine the main bioactive components responsible for the clinical effects. We should not consider the platelet itself nor its growth factors, but certainly the synergy of both. Regarding the tendon healing, the in vivo degranulation of platelets is thought to be critical for the progressive release of growth factors and bioactive proteins. Thus, the capacity of our preparation method to preserve maximal amount of resting and activable platelets may be a specific advantage in such a context of sport regenerative medicine. Consistently, the method for PRP preparation proposed in this study allows high levels of PDGF-AB, VEGF, and TGF- $\beta 1$ release after platelet 

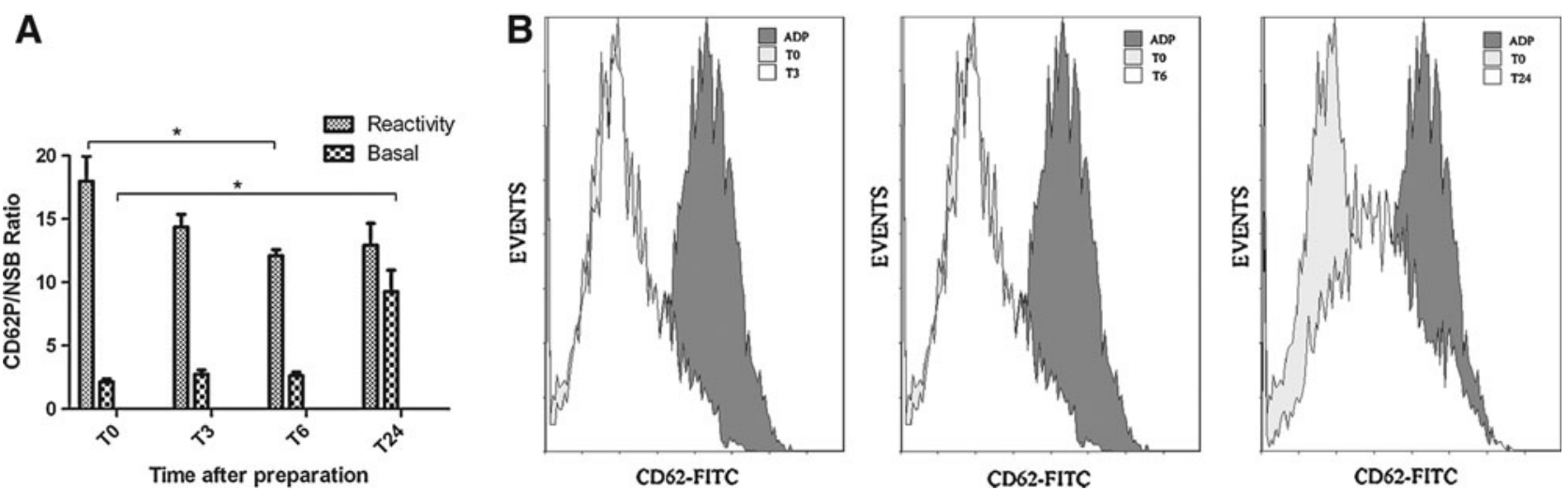

FIG. 7. (A) The spontaneous binding of FITC-conjugated CD62P (basal) to platelets. T0, unstored PRP preparation; T3, storage period of $3 \mathrm{~h}$; T6, $6 \mathrm{~h}$; and T24, storage period of $24 \mathrm{~h}$. The binding of CD62P-FITC to platelets induced by ADP (reactivity). Data obtained for different five donors. ${ }^{*} p<0.008$. (B) Representative flow cytometry histograms. FITC, fluorescein isothiocyanate.

activation by $\mathrm{CaCl}$ and glass tubes. This method is not the most frequently used in the literature, but we also observed that platelet degranulation obtained by successive freeze/ thaw cycles and ultracentrifugation did not permit an optimal detection of VEGF release capacity (data not shown). Our data highlighted the wide heterogeneity of growth factors release by PRP, suggesting a high interindividual variability in cellular production and storage of cytokines. Although the comparison is difficult due to the few available studies and the lack of standardized method, the growth factor concentrations in our experiments appeared to be in the same order of magnitude compared to previous reports. ${ }^{4,18}$ Notably, these results were obtained with a lower centrifugation speed presumed to ensure better platelet functionality. Interestingly, correlation between platelets counts and growth factor release could be observed for PDGF-AB, but not for VEGF and TGF- $\beta 1$. Similar observations were reported by Weibrich et al., ${ }^{19}$ indicating that the platelet count may not be a sufficient marker to predict biological activity. Therefore, there is no simple procedure currently available to obtain a preoperative estimation of the content of individual growth factors in PRP. This information would be helpful to ensure a reliable and reproducible use of PRP for clinical treatment, since the regenerative potential of PRP undoubtedly depends on its growth factor levels. ${ }^{18}$ Thus, the development of new strate- gies allowing a preinjection testing of PRP biological activity is challenging.

With a view to preparing PRP for clinical use, a precise knowledge of storage impact is also mandatory. As suggested by Ref., ${ }^{8}$ measurement of P-selectin membrane levels and quantification of growth factor release are reliable tools for definition of the maximal storage duration for PRP. Increased P-selectin expression during PRP storage has been reported. ${ }^{8}$ In addition, platelets reactivity toward different agonists significantly decreases during storage of platelet concentration or apheresis platelets. ${ }^{20}$ Our results are consistent with these previous reports. We demonstrated that a 3-h storage duration may be compatible with PRP stability. At this time, P-selectin upregulation in response to ADP was maintained at comparable levels compared to unstored PRP. We also demonstrated that growth factor release was unaffected over a period of $6 \mathrm{~h}$ postpurification.

Characterization of PRP prepared by $250-g$ centrifugation also evidenced the presence of residual leukocytes, indicating that one potential limitation of such low-speed protocol is to reduce the efficiency of separation between the different cell components of the buffy coat. It has been suggested that leukocytes from platelet concentrates may participate in protease-dependent proinflammatory effects. ${ }^{4}$ However, their involvement in PRP side effects is currently not
FIG. 8. Growth factor levels in PRP and PPP during storage. T0, unstored PRP preparation; $\mathrm{T} 3$, storage period of $3 \mathrm{~h}$; T6, $6 \mathrm{~h}$; and T24, storage period of $24 \mathrm{~h}$. Data obtained for different three donors. ${ }^{* * *} p<0.0001$.
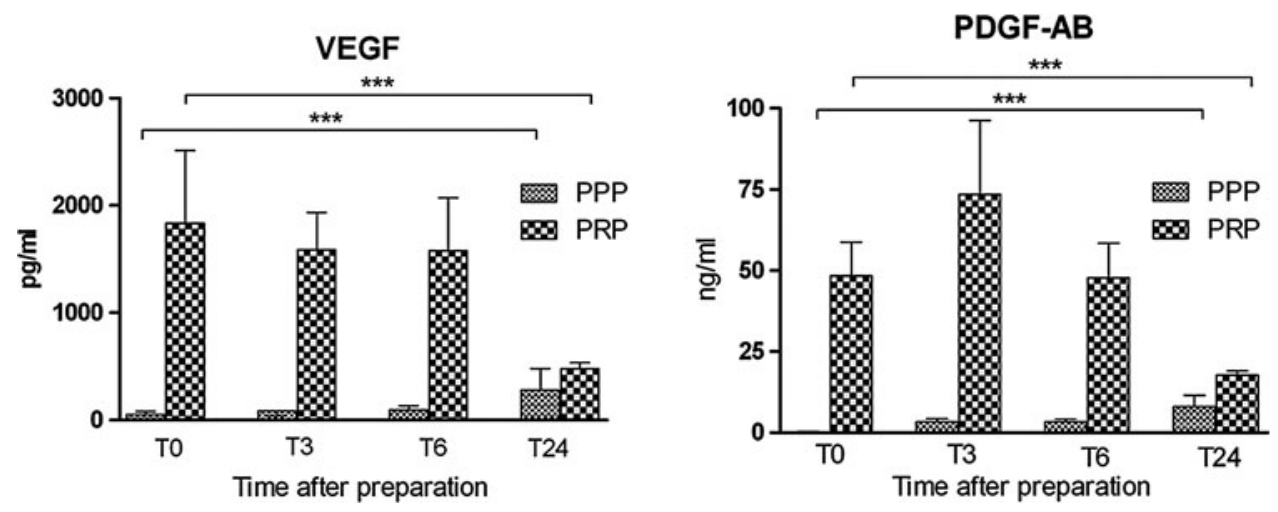
established. Up to now, no undesirable inflammatory reactions have been observed with leukocyte-rich PRP, even in immune-sensitive applications. ${ }^{12}$ Conversely, several studies showed that leukocytes in PRP support antimicrobial effects. ${ }^{21}$ In addition, the counting of residual leukocytes is an important PRP specification, since they contain some growth factors that can modify the growth factor balance. The part of the PDGF-AB supplied by PRP may originate from leukocytes in addition to platelets. ${ }^{18}$ Further investigations are needed to determine the relevance of leukocytes in PRP. ${ }^{12}$

\section{Conclusion}

We propose a simple, fast, and reproductive PRP preparation method based on two successive centrifugations, allowing optimal platelet enrichment, platelet quality preservation, and growth factor release upon in situ activation. Characterization and standardization of the biological properties of PRP form the basis for future clinical studies and are critical steps toward the full definition of PRP clinical values.

\section{Author Disclosure Statement}

The authors would like to make clear that that any conflict of interest, including any that could arise from financial or personal relationships with other people or organizations and inappropriately bias their work, is fully excluded.

\section{References}

1. Gosens T, Peerbooms JC, van Laar W, et al. Ongoing positive effect of platelet-rich plasma versus corticosteroid injection in lateral epicondylitis: a double-blind randomized controlled trial with 2-year follow-up. Am J Sports Med. 2011;39:12001208.

2. Peerbooms JC, Sluimer J, Bruijn DJ, et al. Positive effect of an autologous platelet concentrate in lateral epicondylitis in a double-blind randomized controlled trial: platelet-rich plasma versus corticosteroid injection with a 1-year followup. Am J Sports Med. 2010;38:255-262.

3. Mishra A, Pavelko T. Treatment of chronic elbow tendinosis with buffered platelet-rich plasma. Am J Sports Med. 2006; 34:1774-1778.

4. Anitua E, Sanchez M, Zalduendo MM, et al. Fibroblastic response to treatment with different preparations rich in growth factors. Cell Prolif. 2009;42:162-170.

5. Pignatelli P, Pulcinelli FM, Ciatti F, et al. Acid citrate dextrose (ACD) formula A as a new anticoagulant in the measurement of in vitro platelet aggregation. J Clin Lab Anal. 1995;9:138-140.

6. Slichter SJ, Harker LA. Preparation and storage of platelet concentrates. II. Storage variables influencing platelet viability and function. Br J Haematol. 1976;34:403-419.

7. Luddington RJ. Thrombelastography/thromboelastometry. Clin Lab Haematol. 2005;27:81-90.

8. Tynngard N. Preparation, storage and quality control of platelet concentrates. Transfus Apher Sci. 2009;41:97-104.

9. Gutensohn K, Geidel K, Kroeger N, et al. Platelet function testing in apheresis products: flow cytometric, resonance thrombographic (RTG) and rotational thrombelastographic (roTEG) analyses. Transfus Apher Sci. 2002;26:147-155.

10. Alonso-Escolano D, Strongin AY, Chung AW, et al. Membrane type-1 matrix metalloproteinase stimulates tumour cell-induced platelet aggregation: role of receptor glycoproteins. Br J Pharmacol. 2004;141:241-252.

11. Radomski A, Jurasz P, Alonso-Escolano D, et al. Nanoparticle-induced platelet aggregation and vascular thrombosis. Br J Pharmacol. 2005;146:882-893.

12. Ehrenfest DM, Bielecki T, Del Corso M, et al. Shedding light in the controversial terminology for platelet-rich products: platelet-rich plasma (PRP), platelet-rich fibrin (PRF), platelet-leukocyte gel (PLG), preparation rich in growth factors (PRGF), classification and commercialism. J Biomed Mater Res A. 2010;95:1280-1282.

13. de Vos RJ, Weir A, van Schie HT, et al. Platelet-rich plasma injection for chronic Achilles tendinopathy: a randomized controlled trial. JAMA. 2010;303:144-149.

14. Cho JM, Lee YH, Baek RM, et al. Effect of platelet-rich plasma on ultraviolet $b$-induced skin wrinkles in nude mice. J Plast Reconstr Aesthet Surg. 2010;641:e31-e39.

15. Jungbluth P, Wild M, Grassmann JP, et al. Platelet-rich plasma on calcium phosphate granules promotes metaphyseal bone healing in mini-pigs. J Orthop Res. 2010;28:14481455.

16. Cardigan R, Turner C, Harrison P. Current methods of assessing platelet function: relevance to transfusion medicine. Vox Sang. 2005;88:153-163.

17. Landesberg R, Roy M, Glickman RS. Quantification of growth factor levels using a simplified method of plateletrich plasma gel preparation. J Oral Maxillofac Surg. 2000;58:297-300; discussion 301.

18. Weibrich G, Kleis WK, Hafner G, et al. Comparison of platelet, leukocyte, and growth factor levels in point-of-care platelet-enriched plasma, prepared using a modified Curasan kit, with preparations received from a local blood bank. Clin Oral Implants Res. 2003;14:357-362.

19. Weibrich G, Kleis WK, Hafner G, et al. Growth factor levels in platelet-rich plasma and correlations with donor age, sex, and platelet count. J Craniomaxillofac Surg. 2002;30:97-102.

20. Apelseth TO, Bruserud $O$, Wentzel-Larsen $T$, et al. In vitro evaluation of metabolic changes and residual platelet responsiveness in photochemical treated and gamma-irradiated single-donor platelet concentrates during long-term storage. Transfusion. 2007;47:653-665.

21. Moojen DJ, Everts PA, Schure RM, et al. Antimicrobial activity of platelet-leukocyte gel against Staphylococcus aureus. J Orthop Res. 2008;26:404-410.

Address correspondence to: Olivier Bausset, $M D$ Cell Therapy Unit, University Hospital "La Conception" 147 Boulevard Baille 13005 Marseille France

E-mail: olivier.bausset@wanadoo.fr 